\title{
Development of the fundamentals of thoracic robotic surgery curriculum
}

\author{
Jeffrey S. Levy ${ }^{1}$, Farid Gharagozloo ${ }^{2}$ \\ ${ }^{1}$ Institute of Surgical Excellence, Washington, DC, USA; ${ }^{2}$ AdventHealth Celebration, Celebration, FL, USA \\ Contributions: (I) Conception and design: JS Levy; (II) Administrative support: None; (III) Provision of study materials or patients: None; (IV) \\ Collection and assembly of data: JS Levy; (V) Data analysis and interpretation: JS Levy; (VI) Manuscript writing: Both authors; (VII) Final approval \\ of manuscript: Both authors. \\ Correspondence to: Jeffrey S. Levy, MD. Institute of Surgical Excellence, Washington, DC, USA. Email: jlevy@casenetwork.com.
}

\begin{abstract}
A great technological revolution in surgery occurred with the introduction of laparoscopic and other minimally invasive procedures, with enormous patient benefits. Robotic-assisted surgery (RAS) is a form of minimally invasive surgery that overcomes some of the limitations of laparoscopic techniques. Until recently, there were few standardized curricula for RAS. The Fundamentals of Robotic (FRS) developed a process through expert consensus conferences to develop an effective and validated curriculum for basic robotic surgery. A specialty specific curriculum for thoracic robotic surgery is also needed. The Fundamentals of Thoracic Robotic Surgery (FTRS) Consensus Conference brought together expert thoracic surgeons to build upon the accomplishments of FRS and incorporate the experiences of thoracic societies and academic institutions to establish a standardized FTRS curriculum for the development and maintenance of specialtyspecific robotic surgical skills. A task deconstruction was completed for the thoracic 'signature' procedure, the lobectomy and training items and potential errors were identified for each step of the procedure. A final outline of the FTRS curriculum was developed during the conference and physical and virtual reality thoracic surgery training models were discussed. Following the conference, the steering committee completed the FTRS curriculum with editorial review from all stakeholders. The FTRS consensus conference followed the validated FRS model but in an accelerated process due to important groundwork set by experts in the FRS consensus conferences. A full online curriculum and supporting psychomotor skills training and team communication has been developed for the lobectomy procedure.
\end{abstract}

Keywords: Thoracic surgery; robotic-assisted; Fundamentals of Robotic Surgery (FRS); curriculum development

Submitted Sep 10, 2020. Accepted for publication Nov 26, 2020.

doi: $10.21037 /$ jtd-2019-rts-02

View this article at: http://dx.doi.org/10.21037/jtd-2019-rts-02

\section{Introduction}

A great technological revolution in surgery occurred with the introduction of laparoscopic and other minimally invasive procedures, with enormous patient benefits. Many randomized trials have demonstrated the benefits of minimally invasive surgery over traditional open surgery (1-4). The use of minimally invasive techniques has now penetrated many specialties, such as general surgery, gynecology, urology, colorectal surgery, thoracic surgery, and others.

Robotic-assisted surgery (RAS) is a form of minimally invasive surgery that is able to overcome some of the limitations of laparoscopic techniques. Some of its benefits of RAS include (5):

* High-definition three-dimensional imaging of the endoscope view that increases depth perception and precision.

* Adjustments of the surgeon's console that provide comfortable ergonomically advantageous positions.

* Elimination of motion reversal seen in laparoscopy.

* Increasing degrees of freedom (DOF) in instrument motion with the addition of a wrist-like joint at the 
end of the end-effector to better facilitate suturing and instrument tying.

* Favorable motion scaling by amplifying data or information to increase (or decrease) the distance the tip of an instrument moves relative to the distance the hand controller moves.

* Stable camera platform without tremor, rotation, or migration.

Until recently, there were few standardized curricula for RAS. The Fundamentals of Robotic Surgery (FRS) is a multi-specialty, proficiency-based curriculum of basic cognitive, psychomotor (technical), and team training and communication skills that was developed to train and assess surgeons to safely and efficiently perform roboticassisted surgery $(6,7)$. To establish this curriculum, a fullcycle development process was utilized to ensure the final curriculum and assessment methods would meet the rigorous requirements of determining proficiency, meeting standards, and possibly even fulfilling certification criteria.

To accomplish this goal, the FRS committee convened almost 80 robotic surgery experts, behavioral psychologists, medical educators, statisticians, and psychometricians. Represented in the clinical subject matter experts (SME) were all of the major surgical specialties in the United States that currently perform robotic-assisted surgical procedures, as well as the Department of Defense, and the VA. These leaders in robotic surgery participated in four consensus conferences that are briefly described below (6).

(I) Outcomes Measures: A prioritized matrix of 25 basic robotic surgery skills, outcome measures and metrics was produced, which served as the core material for development and design of the FRS curriculum.

(II) Curriculum Planning: A previously published expert consensus-driven surgical curriculum template was critically reviewed and adopted for the development of the FRS curriculum (8). An outline for subsequent curriculum development was developed.

(III) Curriculum Development: The FRS curriculum was broken down into 4 parts, including Introduction to Robotic Surgery, Didactic Instructions, Psychomotor Skills, and Team Training and Communication Skills. Online modules were developed for each of these areas. In addition, physical models and virtual reality simulation models were developed to train and test psychomotor skills involving seven basic RAS tasks.
(IV) Validation Study Design: The study design was formulated to conduct a formal validation of the didactic online FRS curriculum, psychomotor (technical) skills, and team training and communication skills components. Based on this study design, an international multi-institutional, noninferiority blinded, randomized control trial was conducted that demonstrated effectiveness of the FRS curriculum by demonstrating better performance of those trained using FRS compared with controls.

The Institute for Surgical Excellence (ISE), a 501(c) (3) public non-profit organization dedicated to improving surgical care and patient outcomes, conducted the FRS validation study. ISE also coordinated, facilitated and managed a subsequent Fundamentals of Thoracic Robotic Surgery Consensus Conference that will be described below.

\section{Expert consensus conference}

Based on the groundwork set by the original four FRS consensus conferences, all elements of outcome measures and metrics, curriculum design and early development, simulation design, and team training and communication were all accomplished during a single Fundamentals of Thoracic Robotic Surgery (FTRS) Consensus Conference from February 9-11, 2017.

The goals for the meeting were to:

* Discuss best practices in curriculum development.

* Build upon the accomplishments of FRS and incorporate the experiences of thoracic societies and academic institutions to establish a standardized Thoracic Surgery robotic curriculum for the development and maintenance of specialtyspecific robotic surgical skills.

* Develop a detailed outline for the FTRS training curriculum.

* Design thoracic surgery specific robotic psychomotor skills for the bedside assistant and console surgeon.

* Determine the most appropriate validation process for the curriculum.

During the meeting descriptions from existing thoracic robotic surgery curricula developed by individual institutions including Emory Healthcare, University of Michigan Health System, University of North Carolina and University of Pittsburgh Medical Center were presented. 
Table 1 Lobectomy task deconstruction

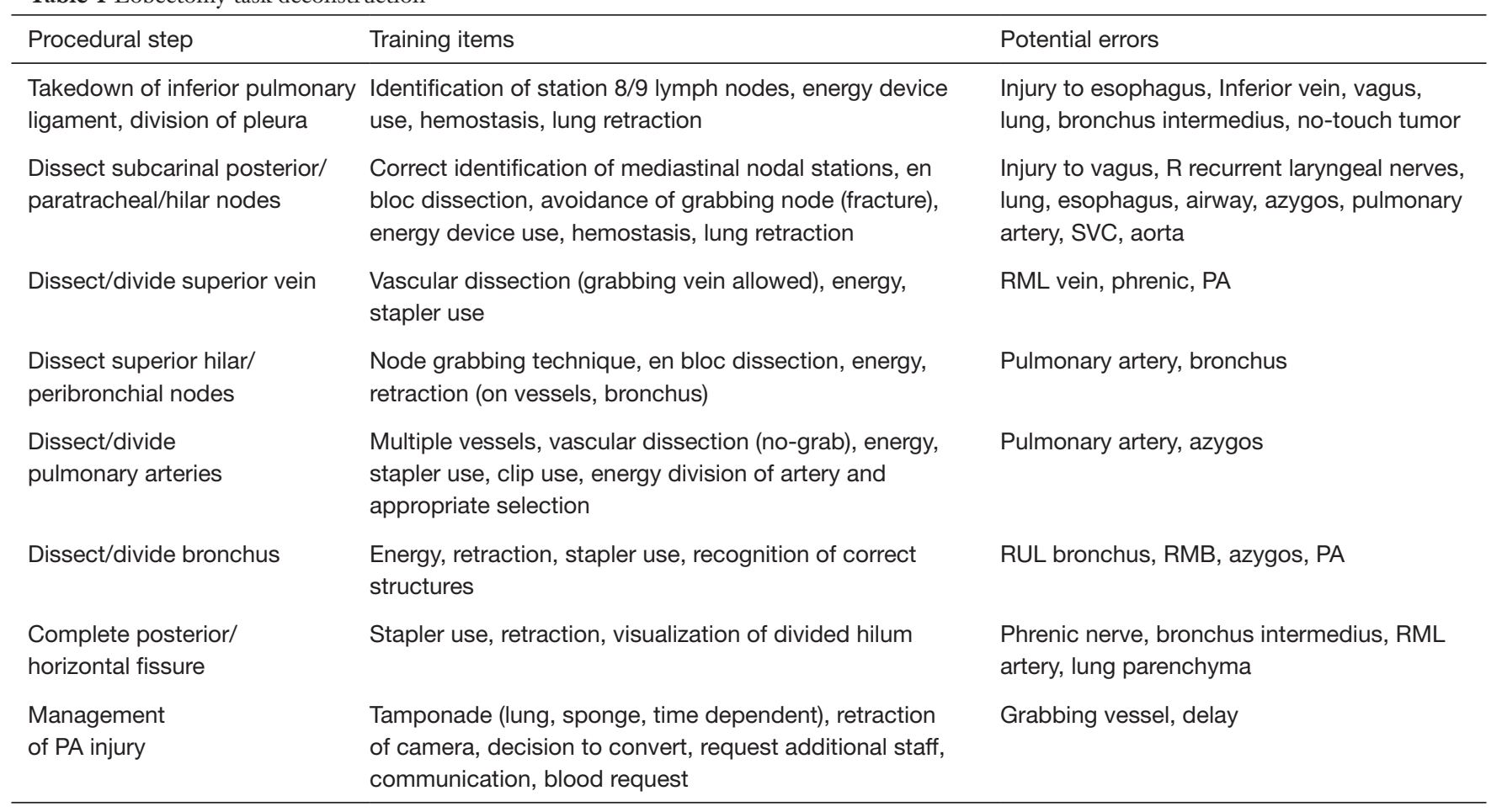

An update was also provided regarding a European thoracic surgery committee that was tasked with development of a robotic curriculum. Commonalities and differences between the various curricula were recorded and discussed.

Next, a task deconstruction was completed for the thoracic 'signature' procedure, the lobectomy, which included:

(I) Takedown of inferior pulmonary ligament, division of pleura.

(II) Dissect subcarinal posterior/paratracheal/hilar nodes.

(III) Dissect/divide superior vein.

(IV) Dissect superior hilar/peribronchial nodes.

(V) Dissect/divide pulmonary arteries.

(VI) Dissect/divide bronchus.

(VII) Complete posterior/horizontal fissure.

(VIII) Management of PA Injury.

For each of these steps, training items and potential errors were identified and are provided in the Table 1.

The consensus conference participants were then divided into two breakout groups responsible for developing the didactic online curriculum and the psychomotor skills curriculum, respectively. The output of the breakout groups included the outline of five modules to teach all components of a lobectomy procedure from the perspective of the pre-procedure patient assessment and preparation, the bedside assistant, the console surgeon, and the operating room team.

\section{Curriculum development}

\section{Curriculum outline}

The final outline of the FTRS curriculum derived from this process is provided below.

\author{
Module 1: introduction \\ * Learning Objectives. \\ * Brief Review of Advantages of Robotic Surgery. \\ * Fundamentals of Thoracic Robotic Surgery (FTRS) \\ Consensus Conference.
}

\section{Module 2: pre-procedure patient assessment and preparation for lobectomy}

* Learning Objectives.

* Pre-Procedure Patient Assessment and Preparation.

- Review of Indications/Contraindications RoboticAssisted Lobectomy.

- Pre-operative Assessment for Robotic-Assisted 
Lobectomy.

- Environment Considerations (Functioning Equipment, Instruments, Suture/Stapling Devices, etc.).

* Module 2 Assessment.

\section{Module 3: bedside assistant}

* Learning Objectives.

* Patient Positioning.

- General Description.

- Lateral Decubitus Position.

- Padding and Securing the Patient.

- Platform Specific Positioning Considerations.

* Port Placement/Trocar Placement.

- General Description.

- Platform Specific Port/Trocar Placement.

* Camera Management.

- Camera Insertion.

- Camera Removal.

- Platform Specific Camera Management.

* Insertion of Instruments.

- General Description.

- Potential Problems When Inserting Instrument.

- Platform Specific Instrument Insertion.

* Instrument Exchange.

- General Description.

* Stapling.

- General Description.

- Staple Height, Length, and Design.

- Stapler Manipulation.

- Platform Specific Stapling.

* Specimen Removal (Nodes, Lobe).

- General Description.

- Platform Specific Specimen Removal.

* Exposure.

- General Description.

* Suctioning.

- General Description.

- Platform Specific Suctioning.

* Standard Undocking.

- General Description.

- Platform Specific Standard Undocking.

* Emergency Undocking.

- General Description.

- Platform Specific Emergency Undocking.

* Postoperative Checklist.

- General Description.

- Platform Specific Postoperative Checklist.
* Summative Assessment.

* Dry Lab Skills Assessment (with Mannequin and Robot).

* Bedside Assistant Certificate.

\section{Module 4: console surgeon}

* Learning Objectives.

* Overview.

* Takedown of Inferior Pulmonary Ligament, Division of Posterior Pleura.

- Anatomy Review.

- Description.

- Instruments Used.

- Video Examples.

- Potential Errors and Complications.

- Dissect Subcarinal Posterior/Paratracheal/Hilar Nodes.

- Anatomy Review.

- Description.

- Instruments Used.

- Video Examples.

- Potential Errors and Complications.

* Dissect/Divide Superior Vein.

- Anatomy Review.

- Description.

- Instruments Used.

- Video Examples.

- Potential Errors and Complications.

- Dissect Superior Hilar/Peribronchial Nodes.

- Anatomy Review.

- Description.

- Instruments Used.

- Video Examples.

- Potential Errors and Complications.

* Dissect/Divide Pulmonary Arteries.

- Anatomy Review.

- Brief Description.

- Instruments Used.

- Video Examples.

- Potential Errors and Complications.

* Dissect/Divide Bronchus.

- Anatomy Review.

- Brief Description.

- Instruments Used.

- Video Examples.

- Potential Errors and Complications.

* Incomplete Posterior/Horizontal Fissure.

- Anatomy Review. 


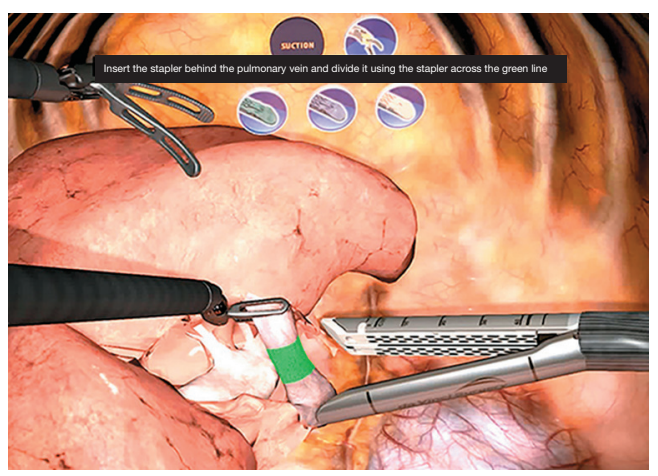

Figure 1 Robotic-assisted lobectomy on the Simbionix RobotiX Mentor from 3D Systems (Permission for use granted by 3D Systems).

- Description.

- Instruments Used.

- Video Examples.

- Potential Errors and Complications.

* Management of Pulmonary Artery Injury.

- Description.

- Instruments Used.

- Video Examples.

- Potential errors and complications.

* Psychomotor Component (Description of Simulation).

- Lung Retraction.

- Dissection of the Pulmonary Artery.

- Stapling and Dividing the Pulmonary Artery.

- Dissection and Removal of Peribronchial Lymph Nodes.

- Managing Minor Bleeding.

* Video Review.

* Psychomotor Skills Assessment.

\section{Module 5: team training}

* Learning Objectives.

* Overview.

* TeamSTEPPS ${ }^{\circledR}$ Background.

* Communication.

- Overview.

- Requests.

- Call-Outs.

- Cross-Checks.

- Check-Backs.

* Situation Awareness.

- Overview.

- Pre-Brief.
- Huddle.

- Debrief.

- SBAR.

* Mutual Support.

- Overview.

- CUS.

- Two-Challenge Rule.

* Checklists.

- WHO Checklist.

- General Pre-operative Phase Checklist.

- Nurse and Operating Department Practitioner (ODP) Checklist.

- Bedside Assistant Related Checklist.

- Trouble Shooting Checklist.

* Leadership.

- Overview.

* Emergency Undocking.

- Principles and Differences from Standard Undocking.

- Deliberate Practice.

* Assessment.

- Cognitive-Questions.

* Robotic Thoracic Surgery Team.

* Team Training Scenarios.

- Pre-Brief Scenario.

- SBAR Scenario.

- CUS Scenario.

- Emergency Undocking.

- Debrief.

\section{Psychomotor skills training}

It was determined at the consensus conference that thoracic surgeons undergoing the FTRS curriculum must complete the online component before progressing to the hands-on psychomotor skills training. At the consensus conference physical thoracic surgery training models (animal tissue and $3 \mathrm{D}$ printed models) were discussed as well as virtual reality simulation models. Following the meeting, several thoracic robotic surgery experts have worked with a simulation company to make the VR lobectomy procedure more realistic and anatomically accurate. An example of the VR lobectomy simulation (developed by 3D Systems) is shown in Figure 1.

\section{Multimedia development}

Following the consensus conference, new medical 


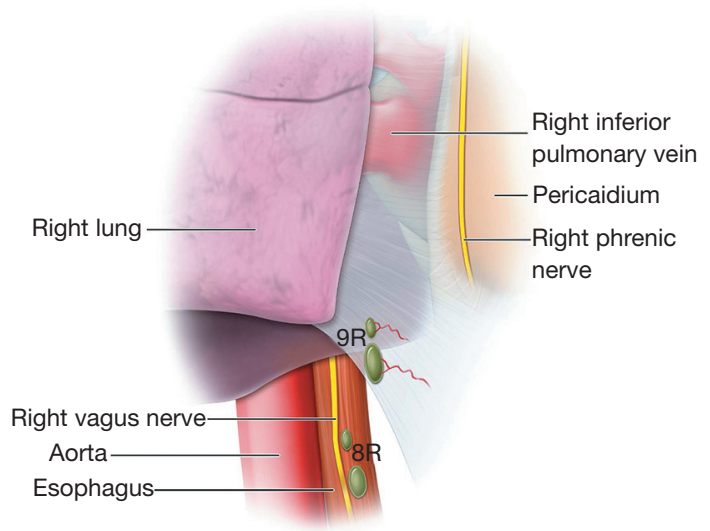

Figure 2 Right inferior pulmonary ligament showing relationship to lung, pericardium, $\mathrm{R}$ phrenic nerve, $\mathrm{R}$ inferior pulmonary vein, esophagus, vagus nerve and diaphragm. Station $8 \mathrm{R}$ and $9 \mathrm{R}$ nodes shown (Permission for use granted by ISE).

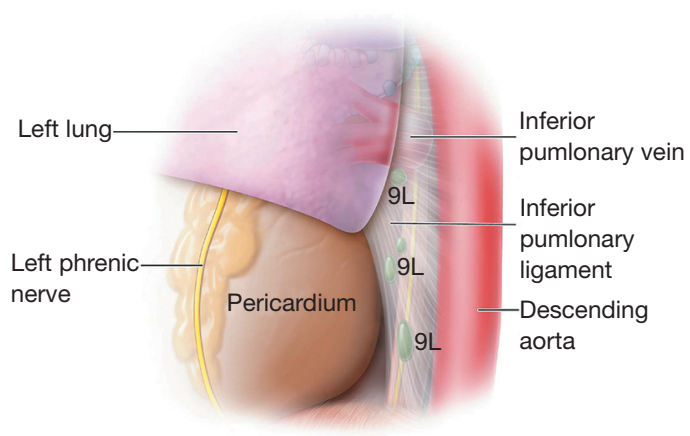

Figure 3 Left inferior pulmonary ligament showing relationship to lung, pericardium, L phrenic nerve, aorta, esophagus/vagus $\mathrm{N}$ and diaphragm. Station $8 \mathrm{R}$ and $9 \mathrm{R}$ nodes shown (Permission for use granted by ISE).

illustrations were developed by ISE and more than two dozen video examples from consensus conference participants to support the curriculum. Some examples are shown (Figures 2-5).

\section{Post-conference action plan}

In addition, a post-conference action plan was initiated and successfully completed including:

(I) Disseminating the information about FTRS to participants' respective organizations and societies to obtain input and "buy in".

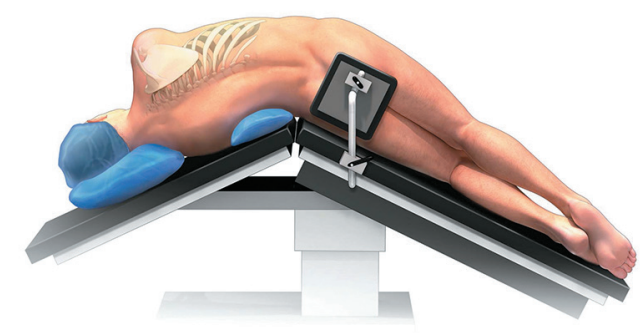

Figure 4 Lateral decubitus, bed flexed at thoracolumbar region to open intercostal spaces as well as to avoid instrumentation approximating lateral pelvis (Permission for use granted by ISE).

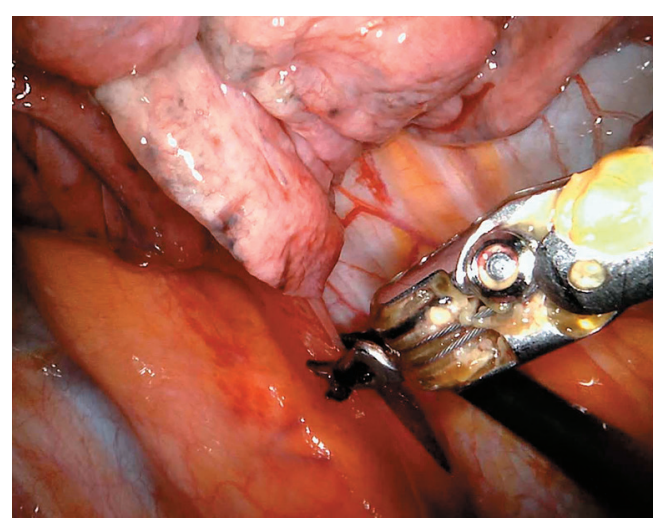

Figure 5 Division of right inferior pulmonary ligament (Permission for use granted by David Rice, MD).

(II) Creating a steering committee comprised of Drs. Anthony Kim, Bernard Park, Allan Pickens and David Rice to finish the content development process for the curriculum.

(III) All participants reviewing and editing the final draft of the FTRS curriculum.

(IV) Collaborating with European thoracic surgeons to coordinate international curriculum development efforts.

\section{Conclusions}

FRS is a proven curriculum development model that included consensus conferences on Outcomes Measures, Curriculum Planning, Curriculum Development, and Validation Study Design. This process led to a validated basic cross-specialty curriculum. The FTRS consensus conference followed this model but in an accelerated 
process due to important groundwork set by experts in the FRS consensus conferences. A full online curriculum and supporting psychomotor skills training and team communication has been developed for the lobectomy procedure. There are plans to duplicate this process for other important thoracic robotic surgical procedures including segmentectomy, esophagectomy, thymectomy, repair of hiatal hernias, Heller myotomy, selective dorsal sympathectomy and first rib resection.

\section{Acknowledgments}

Funding: None.

\section{Footnote}

Provenance and Peer Review: This article was commissioned by the Guest Editor (Ghulam Abbas) for the series "Robotic Thoracic Surgery" published in Fournal of Thoracic Disease. The article has undergone external peer review.

Conflicts of Interest: Both authors have completed the ICMJE uniform disclosure form (available at: http:// dx.doi.org/10.21037/jtd-2019-rts-02). The series "Robotic Thoracic Surgery" was commissioned by the editorial office without any funding or sponsorship. Dr. Levy reports grants from Medtronic, during the conduct of the study. Dr. Gharagozloo reports grants from Medtronic, during the conduct of the study. Both authors have no other conflicts of interest to declare.

Ethical Statement: The authors are accountable for all aspects of the work in ensuring that questions related to the accuracy or integrity of any part of the work are appropriately investigated and resolved.

Open Access Statement: This is an Open Access article distributed in accordance with the Creative Commons
Attribution-NonCommercial-NoDerivs 4.0 International License (CC BY-NC-ND 4.0), which permits the noncommercial replication and distribution of the article with the strict proviso that no changes or edits are made and the original work is properly cited (including links to both the formal publication through the relevant DOI and the license). See: https://creativecommons.org/licenses/by-nc-nd/4.0/.

\section{References}

1. Braga M, Vignali A, Gianotti L, et al. Laparoscopic versus open colorectal surgery: a randomized trial on short-term outcome. Ann Surg 2002;236:759-66; disscussion 767.

2. Nieboer TE, Johnson N, Lethaby A, et al. Surgical approach to hysterectomy for benign gynaecological disease. Cochrane Database Syst Rev 2009;(3):CD003677.

3. Perino A, Cucinella G, Venezia R, et al. Total laparoscopic hysterectomy versus total abdominal hysterectomy: an assessment of the learning curve in a prospective randomized study. Hum Reprod 1999;14:2996-9.

4. Chapron C, Fauconnier A, Goffinet F, et al. Laparoscopic surgery is not inherently dangerous for patients presenting with benign gynecologic pathology: results of a metaanalysis. Hum Reprod 2002;17:1334-42.

5. Schreuder HWR, Verheijen RHM. Robotic surgery. BJOG 2009;116:198-213.

6. Satava RM, Stefanidis D, Levy JS, et al. Proving the Effectiveness of the Fundamentals of Robotic Surgery (FRS) Skills Curriculum: A Single-blinded, Multispecialty, Multi-institutional Randomized Control Trial. Ann Surg 2020;272:384-92.

7. Gallagher AG. Metric-based simulation training to proficiency in medical education:- what it is and how to do it. Ulster Med J 2012;81:107-13.

8. Zevin B, Levy JS, Satava RM, et al. A Consensus-Based Framework for Design, Validation, and Implementation of Simulation-Based Training Curricula in Surgery. J Am Coll Surg 2012;215:580-6.
Cite this article as: Levy JS, Gharagozloo F. Development of the fundamentals of thoracic robotic surgery curriculum. J Thorac Dis 2021;13(10):6116-6122. doi: 10.21037/jtd-2019-rts-02 